Article

\title{
Analytic Automorphisms and Transitivity of Analytic Mappings
}

\author{
Zoriana Novosad ${ }^{1}$ and Andriy Zagorodnyuk ${ }^{2, *}$ (B) \\ 1 Department of Higher Mathematics and Quantitative Methods 10, Lviv University of Trade and Economics, \\ Tuhan-Baranovsky Street, 79005 Lviv, Ukraine; zoryana@lute.lviv.ua \\ 2 Faculty of Mathematics and Computer Science, Vasyl Stefanyk Precarpathian National University, \\ 57 Shevchenka Street, 76018 Ivano-Frankivsk, Ukraine \\ * Correspondence: azagorodn@gmail.com or andriy.zagorodnyuk@pnu.edu.ua
}

Received: 16 November 2020; Accepted: 30 November 2020; Published: 7 December 2020

\begin{abstract}
In this paper, we investigate analytic automorphisms of complex topological vector spaces and their applications to linear and nonlinear transitive operators. We constructed some examples of polynomial automorphisms that show that a natural analogue of the Jacobian Conjecture for infinite dimensional spaces is not true. Also, we prove that any separable Fréchet space supports a transitive analytic operator that is not a polynomial. We found some connections of analytic automorphisms and algebraic bases of symmetric polynomials and applications to hypercyclicity of composition operators.
\end{abstract}

Keywords: topologically transitive operator; hypercyclic operators; function space; analytic functions on Banach spaces; symmetric polynomials on Banach spaces

MSC: 47A16; 47H60; 46E50

\section{Introduction}

Let $X$ be a complex topological vector space. A mapping $F: X \rightarrow X$ is called an analytic automorphism if $F$ is analytic, bijective and $F^{-1}$ is analytic. If both $F$ and $F^{-1}$ are polynomials, then $F$ is a polynomial automorphism. In this paper, we consider polynomial and analytic automorphisms of topological vector spaces and their applications to linear and nonlinear dynamics. We show that in infinite dimension spaces even a triangular polynomial map is not necessary invertible and prove that any separable Fréchet space supports a topologically transitive analytic operator that is not a polynomial. In addition, we found some interesting relations between analytic automorphisms and algebraic bases of symmetric polynomials on Banach spaces and used it for hypercyclic composition operators in spaces of analytic functions.

Let us recall some basic definitions related to analytic mappings of infinitely many variables and chaotic operators.

A function $f$ on a complex topological vector space $X$ with values in the complex plane $\mathbb{C}$ is called analytic if it is continuous and the restriction of $f$ to any finitely dimensional subspace is an analytic function of several variables. If $Y$ is a locally convex topological vector space, then a mapping $F: X \rightarrow Y$ is analytic if $\phi \circ F$ is an analytic function for every continuous linear functional $\phi$ on $Y$. An analytic map $F_{n}$ is an $n$-homogeneous polynomial if $F_{n}(\lambda x)=\lambda^{n} F_{n}(x)$ for all $\lambda \in \mathbb{C}, x \in X$. A finite sum of homogeneous polynomials $F_{0}+F_{1}+\cdots+F_{n}, F_{n} \neq 0$ is a polynomial of degree $n$, where $F_{0}$ is a constant in $Y$. All topological vector spaces considered in this paper are locally convex.

A continuous mapping $T: X \rightarrow X$ is called hypercyclic on a topological vector space $X$ if there is an element $x_{0} \in X$ for which the orbit under $T$, Orb $\left(T, x_{0}\right)=\left\{x_{0}, T x_{0}, T^{2} x_{0}, \ldots\right\}$ is dense in $X$. Every such 
$x_{0}$ is called a hypercyclic element of $T$. A continuous mapping $T: X \rightarrow X$ is called topologically transitive (or just transitive) if for each pair $U, V$ of non-empty open subsets of $X$ there is some $n \in \mathbb{N}$ with $T^{n}(U) \cap V \neq \varnothing$. A transitive map is chaotic if it has a dense set of periodic points. If $X$ is a separable Fréchet space then, according to the Birkhoff transitivity theorem [1] (p. 10), $T$ is topologically transitive if and only if it is hypercyclic.

Let $T$ and $S$ be mapping (not necessary linear) such that $T=F \circ S \circ F^{-1}$ for some continuous bijection $F$. Then $S$ is called conjugate to $T$. Clearly, that the relation of conjugacy is an equivalence relation and preserves hypercyclicity, transitivity and chaoticity. So, if $S$ is a linear transitive (hypercyclic or chaotic) operator and $F$ is an analytic automorphism, we can expect that $T$ is a nonlinear analytic transitive (hypercyclic or chaotic) operator.

The question about existence of hypercyclic polynomials and analytic mappings on Banach and Fréchet spaces were considered in $[2,3]$. Transitive operators on topological vector spaces were studied in [4-7].

Analytic automorphisms can be applied, also, for linear dynamics. For a given analytic map $F: X \rightarrow X$ we denote by $C_{F}$ the composition operator on the space $H(X)$ of all analytic functions on the topological vector space $X$ defined by $C_{F}(f)=f \circ F$. If $C_{\Phi}$ is a hypercyclic composition linear operator on $H(X)$ and $F$ is an analytic automorphism of $X$, then $C_{F \circ \Phi \circ F^{-1}}$ is a hypercyclic composition linear operator which does not commute, in general, with $C_{\Phi}$ [8]. Note that the hypercyclicity of $C_{\Phi}$ on $H(\mathbb{C})$ for $\Phi: x \mapsto x+a$ was proved by Birkhoff in [9] and generalized in [10-12], for the case $H\left(\mathbb{C}^{n}\right)$ and for some infinite dimensional cases in [8,13-15].

Section 1 is devoted to study basic properties of polynomial and analytic automorphisms on infinite dimensional complex spaces. We show that an injective polynomial map $P: \ell_{1} \rightarrow \ell_{1}$ is not necessary an analytic automorphism even if its Jacobi operator $\left(\frac{\partial P_{i}}{\partial x_{j}}\right)$ is a continuous bijection. This gives us a contrast with the finite dimensional case, where the Jacobian Conjecture remains to be unsolved. In addition, we introduce some classes of analytic automorphisms on the space of all complex sequences $\mathbb{C}^{\mathbb{N}}$ and show that subspace

$$
\mathcal{E}=\left\{\left(x_{1}, \ldots, x_{n}, \ldots\right) \in \mathbb{C}^{\mathbb{N}}: \sup _{n}\left|x_{n}\right|^{1 / n}<\infty\right\}
$$

is invariant with respect to this class.

In Section 2 we prove that if a Fréchet space (not necessary separable) admits a linear topologically transitive (chaotic) operator $T$, then $X$ admits a topologically transitive (chaotic) polynomial map of any degree as well as topologically transitive (chaotic) analytic map that is not polynomial. Some examples are constructed.

In Section 3, using some results on symmetric polynomials on $\ell_{1}$, we construct some particular example of "nonlinear weighted backward shift" on $\mathbb{C}^{\mathbb{N}}$ and $\mathcal{E}$, which is transitive.

In Section 4 we prove some applications to linear dynamics. Using the resources in [16] results in the Fréchet algebra of all entire analytic functions $H(\mathcal{E})$ on $\mathcal{E}$ being isometric to the algebra of bounded type symmetric analytic functions $H_{b s}\left(L_{\infty}[0,1]\right)$, we show that the composition operator $f(x) \mapsto f(x+a), f \in H(\mathcal{E})$ is hypercyclic in $H(\mathcal{E})$. Some other hypercyclic composition operators on $H(\mathcal{E})$ and $H_{b s}\left(L_{\infty}[0,1]\right)$ are constructed.

Detailed information about dynamics of linear operators is given in [1,17]. Symmetric analytic functions on $\ell_{p}$ an $L_{p}$ were investigated in [16,18-22]. For details on analytic mappings on topological vector spaces we refer the reader to [23]. 


\section{Analytic Automorphisms of Subspaces of $\mathbb{C}^{\mathbb{N}}$}

A typical example of polynomial automorphism of $\mathbb{C}^{n}$ to itself is a triangular polynomial map $P$ defined by the following way: $P\left(x_{1}, \ldots, x_{n}\right)=\left(y_{1}, \ldots, y_{n}\right)$, where

$$
\begin{aligned}
y_{1} & =c_{1} x_{1}+P_{0} \\
y_{2} & =c_{2} x_{2}+P_{1}\left(x_{1}\right) \\
y_{3} & =c_{3} x_{3}+P_{2}\left(x_{1}, x_{2}\right) \\
& \cdots \\
y_{n} & =c_{n} x_{n}+P_{n-1}\left(x_{1}, \ldots, x_{n-1}\right),
\end{aligned}
$$

where all $c_{1}, \ldots, c_{n}$ are nonzero constants and $P_{j}$ are polynomials on $\mathbb{C}^{j}, j=1, \ldots, n-1$ and $P_{0}=$ const. Note that for the case $\mathbb{C}^{2}$ every polynomial automorphism is a composition of triangular automorphisms, for the case $\mathbb{C}^{3}$ it is not so and for $\mathbb{C}^{n}, n>3$ it is unknown (see [24]). Triangular polynomial automorphisms can be considered in infinite-dimensional spaces. Let $X$ be a complex Banach space which can be represented as a direct topological sum of some nontrivial closed subspaces $X=X_{1} \oplus \cdots \oplus X_{n}$. Then we define $P(x)=P\left(x_{1}, \ldots, x_{n}\right)=\left(y_{1}, \ldots, y_{n}\right), x_{i}, y_{i} \in X_{i}$ by $y_{1}=A_{1}\left(x_{1}\right)$ and $y_{k}=A_{k}\left(x_{k}\right)+P_{k-1}\left(x_{1}, \ldots, x_{k-1}\right), 1 \leq k \leq n$, where $A_{k}$ is a linear isomorphisms of $X_{k}$ to itself and $P_{k-1}: X_{1} \oplus \cdots \oplus X_{k-1}$ is a polynomial. It makes sense to consider the case of an infinity direct sum; however, in this case we have some surprising effects.

Example 1. Let $X=\ell_{p}, 1 \leq p<\infty$ and $\left(e_{k}\right)$ be the standard basis in $\ell_{p}$, that is $e_{n}=(\underbrace{0, \ldots, 0,1}_{k}, 0, \ldots)$.

Let $P: X \rightarrow X$ be a polynomial map such that $y=P(x), x=\sum_{k=1}^{\infty} x_{k} e_{k}$ and

$$
\begin{aligned}
y_{1} & =x_{1} \\
y_{2} & =x_{2}+x_{1}^{2} \\
& \cdots \\
y_{n} & =x_{n}+x_{n-1}^{2}
\end{aligned}
$$

It is easy to see that $P$ is continuous and the inverse map on the range of $P$ can be represented by

$$
\begin{aligned}
x_{1} & =y_{1} \\
x_{2} & =y_{2}-y_{1}^{2} \\
x_{3} & =y_{3}-\left(y_{2}-y_{1}^{2}\right)^{2} \\
& \cdots \\
x_{n} & =y_{n}-\left(y_{n-1}-\left(y_{n-2}-\cdots-\left(y_{2}-y_{1}^{2}\right)^{2} \cdots\right)^{2}\right)^{2} \\
& \cdots
\end{aligned}
$$

The first observation is that $P^{-1}$ is not a polynomial because $\operatorname{deg}\left(x_{n}(y)\right)=2^{n-1}$ for $n>1$. Also, it is interesting that $P$ is not onto. For example, there is no $x \in X$ such that $P(x)=e_{1}$. Indeed, $P^{-1}\left(2 e_{1}\right)=\left(2,-4,8, \ldots,(-1)^{n+1} 2^{n}, \ldots\right) \notin X$. Moreover, the range of $P$ is not dense in $X$. Let us suppose for simplicity that $X=\ell_{1}$. Then the range of $P$ does not contain the ball of radius $r<1 / 4$, centered at $2 e_{1}$. Indeed, if $\left\|y-2 e_{1}\right\|<r$, then $\left|y_{1}\right|>7 / 4$ and $\sum_{k>1}\left|y_{k}\right|<1 / 4$. So, for $n>1$,

$$
\left|x_{n}\right|>49 / 16-1 / 4>2
$$

that is, $x \notin \ell_{1}$. The similar argument works for the general case $\ell_{p}$. 
The famous Jacobian Conjecture asserts that a polynomial map $P: \mathbb{C}^{n} \rightarrow \mathbb{C}^{n}, P(x)=$ $\left(P_{1}(x), \ldots, P_{n}(x)\right)$ is a polynomial automorphism if and only if the Jacobian

$$
\operatorname{det}\left(\frac{\partial P_{i}}{\partial x_{j}}\right)=C \neq 0,
$$

where $C$ is a constant in $\mathbb{C}$. It is easy to check that if $P$ is a polynomial automorphism, then the Jacobian is equal to a nonzero constant. The inverse statement is an open problem for $n \geq 2$. The Jacobian Conjecture was formulated in 1939 by O. H. Keller in [25]. There is a large number of partial results on this conjecture where different approaches are used. In particular, it is known that if a polynomial map $P: \mathbb{C}^{n} \rightarrow \mathbb{C}^{n}$ is injective, then it is surjective and so is a polynomial automorphism. For more details on this open problem, we refer the reader to the survey paper [26].

For the infinite-dimensional case we can replace condition (1) by the following one: for every fixed $x$ the operator $\left(\frac{\partial P_{i}}{\partial x_{j}}\right)$ is invertible; however, Example 1 shows that this condition does not imply that $P$ is a polynomial automorphism. Indeed if $P$ is as in Example 1, then

$$
\left(\frac{\partial P_{i}}{\partial x_{j}}\right)=\left(\begin{array}{cccc}
1 & 0 & 0 & \ldots \\
2 x_{1} & 1 & 0 & \ldots \\
0 & 2 x_{2} & 1 & \ldots \\
\cdots & \ldots & \ldots & \ldots
\end{array}\right)
$$

is invertible but $P$ is not a polynomial automorphism and it is not even an analytic automorphism.

It is interesting to ask about a "natural" domain of a triangular analytic map, that is, the map should be well defined on a topological linear space and invertible. The following proposition is evident.

Proposition 1. A triangular analytic map $F:\left(x_{1}, \ldots, x_{n}, \ldots\right) \mapsto\left(y_{1}, \ldots, y_{n}, \ldots\right)$ of the form

$$
\begin{aligned}
y_{1} & =c_{1} x_{1}+f_{0} \\
y_{2} & =c_{2} x_{2}+f_{1}\left(x_{1}\right) \\
y_{3} & =c_{3} x_{3}+f_{2}\left(x_{1}, x_{2}\right) \\
& \ldots \\
y_{n} & =c_{n} x_{n}+f_{n-1}\left(x_{1}, \ldots, x_{n-1}\right) \\
& \ldots,
\end{aligned}
$$

is well-defined and invertible on the space of all sequences $\mathbb{C}^{\mathbb{N}}$, where $c_{n} \neq 0$ and $f_{n}$ are analytic functions on $\mathbb{C}^{n}, n \in \mathbb{N}$ and $f_{0}=$ const.

Let us consider a partial case of the mapping in Proposition 1, which can be given by a recurrence formula

$$
y_{n}=a_{n 1} x_{n}+a_{n 2} x_{n-1} y_{1}+\cdots+a_{n k} x_{n+1-k} y_{k-1}+\cdots+a_{n n} x_{1} y_{n-1}, \quad n \in \mathbb{N},
$$

where all numbers $a_{n j} \neq 0, j \in \mathbb{N}$.

Proposition 2. There are constants $b_{i_{1} \ldots i_{n}} \in \mathbb{C}, n \in \mathbb{N}$ such that the map (2) can be written by

$$
y_{n}=\sum_{i_{1}+2 i_{2}+\cdots+n i_{n}=n} b_{i_{1} \ldots i_{n}} x_{1}^{i_{1}} \cdots x_{n}^{i_{n}}
$$


Moreover, if $\left|a_{i j}\right| \leq c$ for some positive $c$ and all $i, j \in \mathbb{N}$, then

$$
\left|y_{n}\right| \leq \sum_{k=1}^{n} c^{k} \max _{i \leq n}\left|x_{i}\right|^{k}
$$

Proof. For $n=1$ we have $b_{1}=a_{11}$. Let us suppose that the proposition is true for every $k<n$. Then

$$
y_{n}=\sum_{k=1}^{n} a_{n k} x_{n+1-k} \sum_{i_{1}+\ldots+(k-1) i_{k-1}=k-1} b_{i_{1} \ldots i_{k-1}} x_{1}^{i_{1}} \cdots x_{k-1}^{i_{k-1}} .
$$

Since $i_{1}+\ldots+(k-1) i_{k-1}+n+1-k=k-1+n+(k-1)=n$ for all $k<n$, the vector $y_{n}$ is of the form (3). Also, from (2) we can see that

$$
\left|y_{n}\right| \leq c \max _{i \leq n}\left|x_{i}\right|\left(\sum_{k=1}^{n-1} c^{k} \max _{i \leq n}\left|x_{i}\right|^{k}+1\right)=\sum_{k=1}^{n} c^{k} \max _{i \leq n}\left|x_{i}\right|^{k} .
$$

Corollary 1. Let $F$ be an analytic map on $\mathbb{C}^{\mathbb{N}}$ as in Proposition 2 and $\left|a_{i j}\right| \leq c$ for some positive $c$ and all $i, j \in \mathbb{N}$. If there is a constant $d>0$ such that

$$
\sup _{n \in \mathbb{N}}\left|x_{n}\right|^{1 / n} \leq d
$$

then there is a constant $b>0$ such that

$$
\sup _{n \in \mathbb{N}}\left|y_{n}\right|^{1 / n} \leq b
$$

Proof. From Proposition 2 we have

$$
\sup _{n \in \mathbb{N}}\left|y_{n}\right|^{1 / n} \leq \sup _{n \in \mathbb{N}}\left(\sum_{k=1}^{n} c^{k} \max _{i \leq n}\left|x_{i}\right|^{k}\right)^{1 / n} .
$$

If $c d=1$, then $\sup _{n \in \mathbb{N}}\left|y_{n}\right|^{1 / n} \leq \sup _{n \in \mathbb{N}} n^{1 / n}<2$. In the general case we can compute the sum of the geometric series

$$
\sup _{n \in \mathbb{N}}\left|y_{n}\right|^{1 / n} \leq \sup _{n \in \mathbb{N}}\left(\frac{c^{n} d^{n}-1}{c d-1}\right)^{1 / n}<\infty
$$

because

$$
\limsup _{n \rightarrow \infty}\left(\frac{c^{n} d^{n}-1}{c d-1}\right)^{1 / n}=c d
$$

Let us denote $\mathcal{E}=\left\{x \in \mathbb{C}^{\mathbb{N}}: \sup _{n}\left|x_{n}\right|^{1 / n}<\infty\right\}$.

Theorem 1. Let $F$ be an analytic map on $\mathbb{C}^{\mathbb{N}}$ as in Proposition 2 and there are positive constants $c$ and $c^{\prime}$ such that $\left|a_{i j}\right| \leq c$ and $\left|a_{i 1}\right|^{-1} \leq c^{\prime}, i, j \in \mathbb{N}$. Then $\mathcal{E}$ is F-invariant in $\mathbb{C}^{\mathbb{N}}$ and the restriction $F_{0}$ of $F$ onto $\mathcal{E}$ is a bijection.

Proof. From Corollary 1 , we obtain that the map $F_{0}: \mathcal{E} \rightarrow \mathcal{E}$ is into. The inverse map on the range of $F_{0}$ can be written by

$$
x_{n}=-\frac{1}{a_{n 1}}\left(-y_{n}+a_{n 2} x_{n-1} y_{1}+\cdots+a_{n k} x_{n+1-k} y_{k-1}+\cdots+a_{n n} x_{1} y_{n-1}\right), \quad n \in \mathbb{N}
$$


Since $\left|a_{n 1}\right|^{-1} \leq c^{\prime}$, we can apply to this map Corollary 1 . So, the range of $F_{0}$ is $\mathcal{E}$ and $F_{0}$ is onto. Thus $F_{0}$ is a bijection.

Note that $\mathcal{E}$ admits function space representations. For every $x=\left(x_{1}, \ldots, x_{n}, \ldots\right)$ we can assign a complex function $f_{x}(t), t \in \mathbb{C}$ by

$$
f_{x}(t)=\sum_{n=1}^{\infty} x_{n} t^{n-1}
$$

It is well known that the map $x \mapsto f_{x}$ is a linear bijection from $\mathcal{E}$ to the space $H\left(0_{\mathbb{C}}\right)$ of holomorphic germs at the origin in $\mathbb{C}$. Also, $H\left(0_{\mathbb{C}}\right)$ is isomorphic to the space of functions of exponential type, $H_{\exp }(\mathbb{C})$ via the Borrel transform

$$
f_{x}(t) \mapsto \sum_{n=1}^{\infty} \frac{x_{n} t^{n-1}}{(n-1) !}
$$

Example 2. Let us consider a special case of mapping (2) on $\mathbb{C}^{\mathbb{N}}$. We denote by $\Theta: \mathbb{C}^{\mathbb{N}} \rightarrow \mathbb{C}^{\mathbb{N}}, \Theta(x)=y$ such that

$$
y_{n}=x_{1} y_{n-1}-x_{2} y_{n-2}+\cdots+(-1)^{n-1} x_{n}, \quad n \in \mathbb{N}, x_{0}=y_{0}=0 .
$$

Then $\Theta^{-1}=\Theta$, that is, $\Theta^{2}(x)=x, x \in \mathbb{C}^{\mathbb{N}}$ and $\Theta$ satisfies conditions of Theorem 1 .

\section{The Existence of Hypercyclic Analytic Mappings}

Theorem 2. Let $X$ be an infinite dimension Fréchet space (not necessary separable). If $X$ admits a linear topologically transitive (chaotic) operator $T$, then $X$ admits a topologically transitive (chaotic) polynomial map of any degree as well as topologically transitive (chaotic) analytic map that is not polynomial and is conjugate to $T$.

Proof. It is enough to construct an analytic automorphism $\Phi: X \rightarrow X$ such that $\Phi \circ T \circ \Phi^{-1}$ is nonlinear. We know that there are a lot of ways to define an analytic automorphism. Let $X_{1}$ be a 1-dimensional subspace in $X$ and $X_{2}$ be a complemented subspace, that is, $X=X_{1} \oplus X_{2}$. Let $F$ be an analytic map on $X$ such that $F\left(x_{1}+x_{2}\right)=F\left(x_{1}\right) \in X_{2}$ for all $x_{1} \in X_{1}$ and $x_{2} \in X_{2}$. We set

$$
\Phi=I+F, \quad \text { where } I \text { is the identity operator. }
$$

Then $\Phi^{-1}=I-F$ and so $\Phi$ is an analytic automorphism. Let us write $T(x)=(T(x))_{1}+(T(x))_{2^{\prime}}$ where $(T(x))_{1} \in X_{1}$ and $(T(x))_{2} \in X_{2}$. Then, for every $x=\left(x_{1}, x_{2}\right)=x_{1}+x_{2}, x_{i} \in X_{i}$

$$
\begin{gathered}
\Phi \circ T \circ \Phi^{-1}\left(x_{1}, x_{2}\right)=\Phi \circ T\left(x_{1}, x_{2}-F\left(x_{1}\right)\right) \\
=\Phi\left(\left(T\left(x_{1}, x_{2}-F\left(x_{1}\right)\right)\right)_{1},\left(T\left(x_{1}, x_{2}-F\left(x_{1}\right)\right)\right)_{2}\right) \\
=\left(T\left(x_{1}, x_{2}-F\left(x_{1}\right)\right)\right)_{1}+\left(T\left(x_{1}, x_{2}-F\left(x_{1}\right)\right)\right)_{2}+F\left(\left(T\left(x_{1}, x_{2}-F\left(x_{1}\right)\right)\right)_{1}\right) \\
=T(x-F(x))+F(T(x)-F(x))=T(x)+T \circ F(x)-F \circ T(x)
\end{gathered}
$$

since $F(x) \in X_{2}$ and by the definition of $F, F(T(x)-F(x))=F(T(x))$. Thus, to make sure that $\Phi \circ T \circ \Phi^{-1}$ ia an $m$-degree polynomial we have to guaranty that $T \circ F-F \circ T$ is so. Let us suppose that $T\left(X_{1}\right) \subset X_{2}$ and $F\left(t z_{1}\right)=f(t) z_{2}$ for some $z_{1} \in X_{1}, z_{1} \neq 0$ and $z_{2} \in X$ such that $T\left(z_{2}\right) \neq 0$, where $f$ is a function of $t \in \mathbb{C}$. Then

$$
\Phi \circ T \circ \Phi^{-1}\left(t z_{1}\right)=T\left(t z_{1}\right)+T \circ F\left(t z_{1}\right)-F \circ T\left(t z_{1}\right)=t T\left(z_{1}\right)+f(t) T\left(z_{2}\right) .
$$

Therefore, if $f$ is an $m$-degree polynomial or an analytic function which is not a polynomial, then $\Phi \circ T \circ \Phi^{-1}$ is so. 
Note first that if we put instead of $f$ a continuous function on $\mathbb{C}$ which is not analytic, we can get a continuous nonanalytic topologically transitive (or chaotic) map. Also, $\left(\Phi \circ T \circ \Phi^{-1}\right)^{n}=\Phi \circ T^{n} \circ \Phi^{-1}$, and for the map in Theorem 2,

$$
\left(\Phi \circ T \circ \Phi^{-1}\right)^{n}(x)=T^{n}(x)+T^{n} \circ F(x)-F \circ T^{n}(x) .
$$

It is known (see e.g., [6]) that the left backward shift $T:\left(x_{1}, \ldots, x_{n}, \ldots\right)=\left(x_{2}, \ldots, x_{n+1}, \ldots\right)$ on $\mathbb{C}^{\mathbb{N}}$ is hypercyclic.

Corollary 2. Let $F: \mathbb{C}^{\mathbb{N}} \rightarrow \mathbb{C}^{\mathbb{N}}$ be as in Proposition 1. Then $F \circ T \circ F^{-1}$ is hypercyclic. If the function $f_{1}$ is nonlinear, then $F \circ T \circ F^{-1}$ is nonlinear and if $f_{1}$ is an analytic function which is not a polynomial, then $F \circ T \circ F^{-1}$ is so.

Proof. The hypercyclisity of $F \circ T \circ F^{-1}$ it follows from the hypercyclisity of $T$ and Proposition 1 . Let

$$
\left(z_{1}, \ldots, z_{n}, \ldots\right)=F \circ T \circ F^{-1}\left(x_{1}, \ldots, x_{n}, \ldots\right)
$$

Then from the direct calculations we can see that

$$
z=\frac{c_{1}}{c_{2}}\left(x_{2}-f_{1}\left(\frac{x_{1}}{c_{1}}-f_{0}\right)\right)-f_{0}
$$

that completes the proof.

\section{Analytic Automorphisms and Symmetric Polynomials}

In this section we apply some results on symmetric polynomials of infinite many variables to analytic automorphism. For us it is convenient consider polynomials defined on the complex Banach space $\ell_{1}$. Let us recall some definitions.

A polynomial $P: \ell_{1} \rightarrow \mathbb{C}$ is said to be symmetric if it is invariant with respect to permutations of basis vectors $e_{n}, n \in \mathbb{N}$. Let us denote by $\mathcal{P}_{S}\left(\ell_{1}\right)$ the space of all symmetric polynomials on $\ell_{1}$. It is well known [20] that polynomials

$$
F_{k}(z)=\sum_{n=1}^{\infty} z_{n}^{k}, \quad z=\sum_{n=1}^{\infty} z_{n} e_{n} \in \ell_{1}
$$

form an algebraic basis in $\mathcal{P}_{s}\left(\ell_{1}\right)$, that is, every polynomial in $\mathcal{P}_{s}\left(\ell_{1}\right)$ can be uniquely represented as a finite algebraic combination of polynomials $\left\{F_{k}\right\}_{k=1}^{\infty}$.

If $\left\{Q_{k}\right\}_{k=1}^{\infty}$ is another algebraic basis of homogeneous polynomials in $\mathcal{P}_{s}\left(\ell_{1}\right)$, then

$$
Q_{n}=a_{n 1} F_{n}+a_{n 2} F_{n-1} Q_{1}+\cdots+a_{n k} F_{n+1-k} Q_{k-1}+\cdots+a_{n n} F_{1} Q_{n-1}, \quad n \in \mathbb{N},
$$

where all numbers $a_{n j} \neq 0, j \in \mathbb{N}$. Comparing this equation with (2), we can see that there is a bijection between algebraic bases of $\mathcal{P}_{\mathcal{S}}\left(\ell_{1}\right)$ and analytic automorphisms (2) on $\mathbb{C}^{\mathbb{N}}$. We denote by $F_{Q}$ the map (2) associated with (4).

The basis $\left\{F_{k}\right\}$ is called the basis of power polynomials. There some other classical algebraic basis in $\mathcal{P}_{S}\left(\ell_{1}\right)$, so-called the basis of elementary symmetric polynomials

$$
G_{k}(z)=\sum_{n_{1}<n_{2}<\cdots<n_{k}} z_{n_{1}} \ldots z_{n_{k}} .
$$

By the Newton formulas, the corresponding analytic automorphism $F_{G}$ can be written as

$$
n y_{n}=\sum_{j=1}^{n}(-1)^{j+1} x_{j} y^{n-j}, \quad x, y \in \mathbb{C}^{\mathbb{N}}
$$


In [8] it is proved that if $\tau_{a}$ is a homomorphism of $\mathcal{P}_{s}\left(\ell_{1}\right)$, defined on the algebraic basis $\left\{F_{k}\right\}$ by

$$
F_{k}(z) \mapsto F_{k}(z)+F_{k}(a)
$$

for some $a \in \ell_{1}, a \neq 0$, then

$$
\tau_{a}\left(G_{n}\right)(z)=\sum_{j=0}^{n} G_{j}(z) G_{n-j}(a), \quad z \in \ell_{1}, n \in \mathbb{N}, G_{0}=1 .
$$

Proposition 3. Let $y=F_{G}(x)$ and $y^{\prime}=F_{G}\left(x^{\prime}\right)$ for some $x, x^{\prime} \in \mathbb{C}^{\mathbb{N}}$. Then

$$
u=F_{G}\left(x+x^{\prime}\right), \quad \text { where } u_{n}=\sum_{j=0}^{n} y_{j} y_{n-j}^{\prime}, \quad y_{0}=y_{0}^{\prime}=1 .
$$

Proof. If there are $z, a \in \ell_{1}$ such that $F_{n}(z)=x_{n}$ and $F_{n}(a)=x_{n}^{\prime}$ for all natural $n$, then $G_{n}(z)=y_{n}$ and $G_{n}(a)=y_{n}^{\prime}$, and the statement is true by Formula (5). Let us show that it is true for all $x, x^{\prime} \in \mathbb{C}^{\mathbb{N}}$.

Let $u=F_{G}\left(x+x^{\prime}\right)$ and we suppose for contrary that $u \neq v$, where

$$
v_{n}=\sum_{j=0}^{n} y_{j} y_{n-j}^{\prime}
$$

Let $m$ be the smallest natural number such that $u_{m} \neq v_{m}$. We denote by $w$ and $b$ vectors in $\ell_{1}$ such that $F_{n}(w)=x_{n}$ and $F_{n}(b)=x_{n}^{\prime}$ for $1 \leq n \leq m$. Such vectors always exist (see e.g., [18]). Then $G_{n}(w)=y_{n}$ for $1 \leq n \leq m$ because $y_{n}$ depends only on $x_{1}, \ldots, x_{n}$. By the same reason, $G_{n}(a)=y_{n}^{\prime}$ for $1 \leq n \leq m$. So, according to (5), $u_{m}=v_{m}$. A contradiction.

In [5], the following result was proved.

Theorem 3. Let $X$ be a locally convex sequence space such that $c_{00} \subset X \subset \mathbb{C}^{\mathbb{N}}$ with continuous inclusions, and such that $c_{00}$ is dense in $X$. If $\omega$ is a strictly positive weight such that the weighted backward shift $T_{\omega}$ is continuous on $X$, then $I+T_{\omega}$ is a transitive operator on $X$.

Corollary 3. Let $\omega$ be a strictly positive weight. The mapping $\Phi: \mathbb{C}^{\mathbb{N}} \rightarrow \mathbb{C}^{\mathbb{N}}$ defined by

$$
\Phi(y)=u, \quad \text { where } u_{n}=\sum_{j=0}^{n} \omega_{n-j} y_{j} y_{n+1-j}, \quad n \in \mathbb{N}
$$

is transitive and so hypercyclic.

Proof. Note that if $x=F_{G}^{-1}(y)$, then

$$
\left(I+T_{\omega}\right) \circ F_{G}^{-1}(y)=x+T_{\omega}(x)=\left(x_{1}+\omega_{1} x_{2}, \ldots, x_{n}+\omega_{n} x_{n+1}, \ldots\right) .
$$

Thus, according to Proposition 3,

$$
\Phi(y)=F_{G} \circ\left(I+T_{\omega}\right) \circ F_{G}^{-1}(y) .
$$

By Theorem 3, $I+T_{\omega}$ is transitive. Hence $\Phi$ is transitive and so hypercyclic, since $\mathbb{C}^{\mathbb{N}}$ is a Fréchet space. 
Let us consider a modified version of $F_{G}$, defined by

$$
v=F(x), \quad v_{n}=\sum_{j=1}^{n-1}(-1)^{n+1} \frac{x_{j} v_{n-j}}{n-j}+(-1)^{n+1} x_{n}, \quad x \in \mathbb{C}^{\mathbb{N}}, n \in \mathbb{N} .
$$

It is easy to see that if we put $y_{n}=v_{n} / n$, then $y=F_{G}(x)$. Using similar arguments as in Proposition 3 we have the following result.

Proposition 4. Let $v=F(x)$ and $v^{\prime}=F\left(x^{\prime}\right)$ for some $x, x^{\prime} \in \mathbb{C}^{\mathbb{N}}$. Then

$$
u=F\left(x+x^{\prime}\right), \quad \text { where } \quad u_{n}=\sum_{j=0}^{n} \frac{n}{j(n-j)} v_{j} v_{n-j}^{\prime}, \quad y_{0}=y_{0}^{\prime}=1
$$

The mapping $F$ is interesting for us because the subspace $\mathcal{E} \subset \mathbb{C}^{\mathbb{N}}$ is invariant of $F$ by Theorem 1 . So, we have the following theorem.

Theorem 4. Let $\omega$ be a strictly positive weight. The mapping $\Psi: \mathbb{C}^{\mathbb{N}} \rightarrow \mathbb{C}^{\mathbb{N}}$ defined by

$$
\Psi(y)=u, \quad \text { where } \quad u_{n}=\sum_{j=0}^{n} \frac{n}{j(n-j+1)} \omega_{n-j} y_{j} y_{n+1-j}, \quad n \in \mathbb{N}
$$

is hypercyclic. If $\omega$ is such that the weighted backward shift $T_{\omega}$ is continuous on $\mathcal{E}$, then the restriction $\Psi_{0}$ of $\Psi$ to $\mathcal{E}$ is transitive.

Proof. As in Corollary 3, we can see that $\Psi=F \circ\left(I+T_{\omega}\right) \circ F^{-1}$ and if $F_{0}$ is the restriction of $F$ to $\mathcal{E}$, then $\Psi=F_{0} \circ\left(I+T_{\omega}\right) \circ F_{0}^{-1}$.

\section{Applications to Linear Dynamics}

If we have an analytic automorphism $F$ on a topological vector space $X$, then the composition operator $C_{F}$ is a linear isomorphism of the space $H(X)$ of analytic functions on $X$, defined by $C_{F}(f)=$ $f \circ F, f \in H(X)$. It is well known that the translation operator $f(x) \mapsto f(x+a)$ defined on the space of entire functions $H\left(\mathbb{C}^{n}\right)$ is hypercyclic if $a \neq 0$. Infinite-dimensional generalizations of this results can be found in $[8,13,15]$.

Let us denote by $H(\mathcal{E})$ the space of all analytic functions on $\mathcal{E}$. In [16], it is proved that $H(\mathcal{E})$ is a Fréchet algebra and isomorphic to algebra $H_{b s}\left(L_{\infty}[0 ; 1]\right)$ of all symmetric analytic functions of bounded type on $L_{\infty}[0 ; 1]$. Let us recall that algebra $H_{b s}\left(L_{\infty}[0 ; 1]\right)$ consists of all analytic functions on $L_{\infty}[0 ; 1]$ which are bounded on bounded subsets and invariant with respect measure-preserving measurable automorphisms of $[0 ; 1]$. This algebra is generated by polynomials

$$
R_{n}(z)=\int_{[0 ; 1]}(z(t))^{n} d t, \quad z(t) \in L_{\infty}[0 ; 1],
$$

that is, every polynomial in $H_{b s}\left(L_{\infty}[0 ; 1]\right)$ is an algebraic combination of polynomials $\left\{R_{n}\right\}$ and the set of polynomials is dense in $H_{b s}\left(L_{\infty}[0 ; 1]\right)$. The topology of $H_{b s}\left(L_{\infty}[0 ; 1]\right)$ is the projective topology generated by norms

$$
\|f\|_{r}=\sup _{\|z\| \leq r}|f(z)|, \quad f \in H_{b s}\left(L_{\infty}[0 ; 1]\right), \quad r \in \mathbb{Q}_{+} .
$$

According to [16], the isomorphism between $H_{b s}\left(L_{\infty}[0 ; 1]\right)$ and $H(\mathcal{E})$ is given by

$$
R_{n} \mapsto \phi_{n}, \quad n \in \mathbb{N},
$$

where $\phi_{n}$ is the coordinate functional, $\phi_{n}(x)=x_{n}, x=\left(x_{1}, \ldots, x_{n}, \ldots\right) \in \mathcal{E}$. 
In [21], it is proved that $H_{b s}\left(L_{\infty}[0 ; 1]\right)$ is isomorphic to the algebra of symmetric analytic functions of bounded type on $L_{\infty}[0 ; \infty) \cap L_{1}[0 ; \infty)$, which is denoted by $H_{b s}\left(L_{\infty}[0 ; \infty) \cap L_{1}[0 ; \infty)\right.$ ). Algebra $H_{b s}\left(L_{\infty}[0 ; \infty) \cap L_{1}[0 ; \infty)\right)$ is generated by polynomials

$$
\mathcal{R}_{n}(z)=\int_{[0 ; \infty)}(z(t))^{n} d t, \quad z(t) \in L_{\infty}[0 ; \infty) \cap L_{1}[0 ; \infty)
$$

and the isomorphism is given by

$$
\mathcal{R}_{n} \mapsto R_{n}, \quad n \in \mathbb{N} .
$$

Let $x=x(t)$ and $y=y(t)$ belong to $L_{\infty}[0, \infty) \cap L_{1}[0, \infty)$. Let us define $z=x \bullet y$ by

$$
z(t)=\left\{\begin{array}{lll}
x(t-n), & \text { if } & 2 n \leq t<2 n+1 \\
y(t-n-1), & \text { if } & 2 n+1 \leq t<2 n+2
\end{array}\right.
$$

$n=0,1,2, \ldots$. Clearly that

$$
\mathcal{R}_{n}(x \bullet y)=\mathcal{R}_{n}(x)+\mathcal{R}_{n}(y), \quad n \in \mathbb{N} .
$$

In [14], it is proved that the operator $f(x) \mapsto f(x \bullet y)$ is hypercyclic in $H_{b s}\left(L_{\infty}[0 ; \infty) \cap L_{1}[0 ; \infty)\right)$ for any fixed $y \in L_{\infty}[0 ; \infty) \cap L_{1}[0 ; \infty), y \neq 0$. In [16], it is shown that for every $c=\left(c_{1}, \ldots, c_{n}, \ldots\right) \in \mathcal{E}$ there is a function $y(t) \in L_{\infty}[0 ; \infty) \cap L_{1}[0 ; \infty)$ such that $\mathcal{R}_{n}(y)=c_{n}, n \in \mathbb{N}$. Combining mentioned results in $[14,16,21]$ we have the following theorem.

Theorem 5. Let $c=\left(c_{1}, \ldots, c_{n}, \ldots\right) \in \mathcal{E}, c \neq 0$. Then operator $\mathcal{T}_{c}$ on $H(\mathcal{E})$

$$
\mathcal{T}_{c}(f(x))=f(x+c)
$$

is hypercyclic.

Let $\left\{\mathcal{Q}_{n}\right\}$ be another algebraic basis of homogeneous polynomials in $L_{\infty}[0 ; \infty) \cap L_{1}[0 ; \infty)$, $\operatorname{deg} \mathcal{Q}_{n}=n$. Then

$$
\mathcal{Q}_{n}=\sum_{k=1}^{n} a_{n k} \mathcal{R}_{n+1-k} \mathcal{Q}_{k-1}
$$

If the mapping $y=F(x)$ defined by

$$
y_{n}=\sum_{k=1}^{n} a_{n k} x_{n+1-k} y_{k-1}
$$

is an analytic automorphism of $\mathcal{E}$, then for every $y=\left(y_{1}, \ldots, y_{n}, \ldots\right)$ there exists $z(t) \in L_{\infty}[0 ; \infty) \cap$ $L_{1}[0 ; \infty)$ such that $y_{n}=\mathcal{Q}_{n}(z), n \in \mathbb{N}$. We denote by $C_{F}$ the composition operator $C_{F}(f)=f \circ F$, $f \in H(\mathcal{E})$. Since $F$ is an analytic automorphism of $\mathcal{E}$, then $C_{F}$ is a continuous isomorphism of $H(\mathcal{E})$.

Corollary 4. Let $\left\{\mathcal{Q}_{n}\right\}$ be an algebraic basis of polynomials in $L_{\infty}[0 ; \infty) \cap L_{1}[0 ; \infty)$, defined by (7) such that the mapping (8) is an analytic automorphism of $\mathcal{E}$. Let $u(t)$ be a function in $L_{\infty}[0 ; \infty) \cap L_{1}[0 ; \infty)$ such that $\mathcal{Q}_{n}(u) \neq 0$ for some $n$. Then the homomorphism of $H_{b s}\left(L_{\infty}[0 ; \infty) \cap L_{1}[0 ; \infty)\right)$ defined on $\left\{\mathcal{Q}_{n}\right\}$ by

$$
\mathcal{Q}_{n} \mapsto \mathcal{Q}_{n}+\mathcal{Q}_{n}(u)
$$

is hypercyclic and invertible. 
Proof. For every $f(y) \in H(\mathcal{E}), y=\left(y_{1}, \ldots, y_{n}, \ldots\right)$ we claim that $f\left(\mathcal{Q}_{1}(z), \ldots, \mathcal{Q}_{n}(z), \ldots\right)$ belongs to $H_{b s}\left(L_{\infty}[0 ; \infty) \cap L_{1}[0 ; \infty)\right)$. Indeed,

$$
\left(\mathcal{Q}_{1}(z), \ldots, \mathcal{Q}_{n}(z), \ldots\right)=F\left(\mathcal{R}_{1}(z), \ldots, \mathcal{R}_{n}(z), \ldots\right)
$$

and so

$$
f\left(\mathcal{Q}_{1}(z), \ldots, \mathcal{Q}_{n}(z), \ldots\right)=C_{F}(f)\left(\mathcal{R}_{1}(z), \ldots, \mathcal{R}_{n}(z), \ldots\right) \in H(\mathcal{E}) .
$$

Since $\mathcal{T}_{c}$ is hypercyclic for any $c \neq 0, C_{F}^{-1} \mathcal{T}_{c} C_{F}$ is hypercyclic. Set $c_{n}=\mathcal{Q}_{n}(u)$ for a function $u(t) \in L_{\infty}[0 ; \infty) \cap L_{1}[0 ; \infty)$ such that $\mathcal{Q}_{n}(u) \neq 0$ for some $n$. Then

$$
f\left(\mathcal{Q}_{1}(z)+\mathcal{Q}_{1}(u), \ldots, \mathcal{Q}_{n}(z)+\mathcal{Q}_{n}(u), \ldots\right)=C_{F}^{-1} \mathcal{T}_{c} C_{F}(f)\left(\mathcal{R}_{1}(z), \ldots, \mathcal{R}_{n}(z), \ldots\right) .
$$

Example 3. Let $F_{0}$ be the restriction of the analytic automorphism (6) to $\mathcal{E}$. That is,

$$
y=F(x), \quad \text { where } \quad y_{n}=\sum_{j=1}^{n-1}(-1)^{n+1} \frac{x_{j} y_{n-j}}{n-j}+(-1)^{n+1} x_{n}, \quad x \in \mathbb{C}^{\mathbb{N}}, n \in \mathbb{N}
$$

and $T_{x^{\prime}}(x)=x+x^{\prime}$. Then, by Proposition 4,

$$
\left(F_{0} \circ T_{x^{\prime}} \circ F_{0}^{-1}(y)\right)_{n}=\sum_{i+j=n, i j \neq 0} \frac{i+j}{i j} y_{j} y_{j}^{\prime}+y_{n}+y_{n}^{\prime} .
$$

Thus $C_{F_{0} \circ T_{x^{\prime}} \circ F_{0}^{-1}(y)}=C_{F_{0}}^{-1} \mathcal{T}_{x^{\prime}} C_{F_{0}}$ is hypercyclic on the Fréchet algebra $H(\mathcal{E})$.

\section{Discussion and Conclusions}

The paper is an invitation to study analytic automorphisms of infinite dimensional topological vector spaces and possible applications in functional analysis. We show that, by using analytic and polynomial automorphisms, it is possible to construct various examples of nonlinear topological transitive mappings. On the other hand, if $F$ is an analytic automorphisms of a topological vector space $X$, then the composition operator $C_{F}$ is an isomorphism of the algebra of analytic functions $H(X)$. Thus, it is possible to construct new hypercyclic composition operators on spaces of analytic functions. Some applications for linear dynamics on spaces of symmetric analytic functions are proposed.

Note that if we use injective analytic maps with dense ranges $F: Y \rightarrow X$, instead of analytic automorphisms, then we can get quasiconjugate mappings $S$ and $T$, that is, $T \circ F=F \circ S$. The quasiconjugacy is more general than conjugacy and also preserves topological transitivity and chaoticity [1] (pp. 11-12). However, there are some technical difficulties in constructing natural examples of transitive analytic mappings by using the quasiconjugacy, because the closure of the range of an analytic mapping is not necessary a linear subspace in $X$. Our future investigations will study this further.

Author Contributions: Z.N. and A.Z. contributed equally to this work. All authors have read and agreed to the published version of the manuscript.

Funding: This research was funded by the National Research Foundation of Ukraine, 2020.02/0025, 0120U103996.

Acknowledgments: The authors are grateful to anonymous referees for important suggestions and guidelines in order to make this paper more coherent.

Conflicts of Interest: The authors declare no conflict of interest. 


\section{References}

1. Grosse-Erdmann K.G.; Peris Manguillot, A. Linear Chaos; Springer: London, UK, 2011. [CrossRef]

2. Bernardes, N.C.; Peris, A. On the Existence of Polynomials with Chaotic Behaviour. J. Funct. Spaces 2013, 2013, 320961. [CrossRef]

3. Peris, A. Chaotic polynomials on Banach spaces. J. Math. Anal. Appl. 2003, 287, 487-493. [CrossRef]

4. Ansari, S. I. Existence of hypercyclic operators on topological vector spaces. J. Funct. Anal. 1997, 148, 384-390. [CrossRef]

5. Bonet, J.; Frerick, L.; Peris, A.; Wengenroth, J. Transitive and hypercyclic operators on locally convex spaces. Bull. Lond. Math. Soc. 2005, 37, 254-264. [CrossRef]

6. Bonet, J.; Peris, A. Hypercyclic Operators on Non-normable Fréchet Spaces. J. Funct. Anal. 1998, 159, 587-595. [CrossRef]

7. Manoussos, A. A Birkhoff type transitivity theorem for non-separable completely metrizable spaces with applications to linear dynamics. J. Oper. Theory 2013, 70, 165-174. [CrossRef]

8. Novosad, Z.; Zagorodnyuk A. Polynomial automorphisms and hypercyclic operators on spaces of analytic functions. Arch. Math. 2007, 89, 157-166. [CrossRef]

9. Birkhoff, G.D. Démonstration d'un théorème élémentaire sur les fonctions entières. CR Acad. Sci. Paris 1929, 189, 473-475.

10. Bourdon, P.S.; Shapiro, J.H. Cyclic phenomena for composition operators. Mem. Amer. Math. Soc. 1997, 125, 1-46. [CrossRef]

11. Chan, K. C.; Shapiro, J. H. The cyclic behavior of translation operators on Hilbert spaces of entire functions. Indiana Univ. Math. J. 1991, 40, 1421-1449. [CrossRef]

12. Godefroy, G.; Shapiro, J.H. Operators with dense, invariant, cyclic vector manifolds. J. Funct. Anal. 1991, 98, 229-269. [CrossRef]

13. Aron R.; Bès, J. Hypercyclic differentiation operators. Contemp. Math. 1999, 232, 39-46.

14. Chernega, I.; Holubchak, O.; Novosad, Z.; Zagorodnyuk, A. Continuity and hypercyclicity of composition operators on algebras of symmetric analytic functions on Banach spaces. Eur. J. Math. 2020, 6, 153-163. [CrossRef]

15. Mozhyrovska, Z. H.; Zagorodnyuk, A. V. Hypercyclic behavior of translation operators on spaces of analytic functions on Hilbert spaces. J. Funct. Spaces 2015, 2015, 139289. [CrossRef]

16. Galindo, P.; Vasylyshyn, T.; Zagorodnyuk, A. Analytic structure on the spectrum of the algebra of symmetric analytic functions on $L_{\infty}$. Rev. Real Acad. Cienc. Exactas Fis. Nat. Ser. A Mat. 2020, 114, 56. [CrossRef]

17. Bayart, F.; Matheron, E. Dynamics of Linear Operators; Cambridge University Press: New York, NY, USA, 2009.

18. Alencar, R.; Aron, R.; Galindo, P.; Zagorodnyuk, A. Algebras of symmetric holomorphic functions on $\ell_{p}$. Bull. Lond. Math. Soc. 2003, 35, 55-64. [CrossRef]

19. Chernega, I.; Galindo, P.; Zagorodnyuk, A. Some algebras of symmetric analytic functions and their spectra. Proc. Edinb. Math. Soc. 2012, 55, 125-142. [CrossRef]

20. González, M.; Gonzalo, R.; Jaramillo, J.A. Symmetric polynomials on rearrangement-invariant function spaces. J. Lond. Math. Soc. 1999, 59, 681-697. [CrossRef]

21. Vasylyshyn, T. Algebras of entire symmetric functions on spaces of Lebesgue-measurable essentially bounded functions. J. Math. Sci. 2020, 246, 264-276. [CrossRef]

22. Chernega, I.; Zagorodnyuk, A. Note on Bases in Algebras of Analytic Functions on Banach Spaces. Carpathian Math. Publ. 2019, 11, 42-47. [CrossRef]

23. Dineen, S. Complex Analysis on Infinite Dimensional Spaces; Springer: Berlin/Heidelberg, Germany, $1999 ;$ p. 543. [CrossRef]

24. Shestakov, I.; Umirbaev, U. The tame and the wild automorphisms of polynomial rings in three variables. J. Am. Math. Soc. 2004, 17, 197-227. [CrossRef]

25. Keller, O.H. Ganze Cremonatransformationen Monatschr. Math. Phys. 1939, 47, 229-306. 
26. van den Essen, A. Polynomial Automorphisms and the Jacobian Conjecture; Birkhäuser: Basel, Switzerland, 2000 ; p. 329.

Publisher's Note: MDPI stays neutral with regard to jurisdictional claims in published maps and institutional affiliations.

(C) 2020 by the authors. Licensee MDPI, Basel, Switzerland. This article is an open access article distributed under the terms and conditions of the Creative Commons Attribution (CC BY) license (http://creativecommons.org/licenses/by/4.0/). 